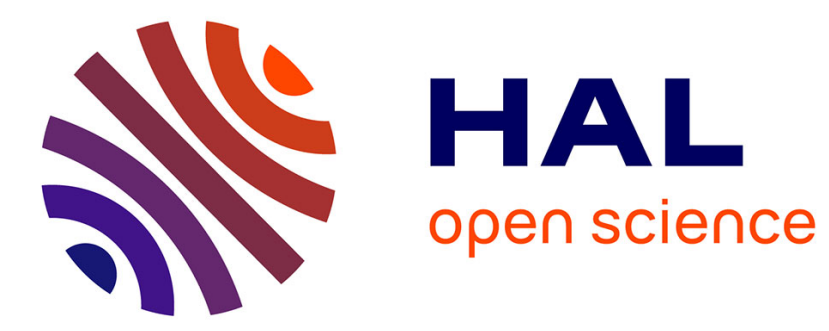

\title{
La notion de personne dans l'Éthique de Spinoza
}

Eric Delassus

\section{To cite this version:}

Eric Delassus. La notion de personne dans l'Éthique de Spinoza. Enseignement Philosophique, 2009, 59 (6), pp.21-37. hal-00596859v2

\section{HAL Id: hal-00596859 \\ https://hal.science/hal-00596859v2}

Submitted on 29 Oct 2013

HAL is a multi-disciplinary open access archive for the deposit and dissemination of scientific research documents, whether they are published or not. The documents may come from teaching and research institutions in France or abroad, or from public or private research centers.
L'archive ouverte pluridisciplinaire HAL, est destinée au dépôt et à la diffusion de documents scientifiques de niveau recherche, publiés ou non, émanant des établissements d'enseignement et de recherche français ou étrangers, des laboratoires publics ou privés. 


\title{
LA NOTION DE PERSONNE
}

\section{DANS L'ÉTHIQUE DE SPINOZA ${ }^{1}$}

\author{
Éric DELASSUS \\ Lycée Jacques Cœur de Bourges \\ \& Université François Rabelais, Tours
}

S'il peut sembler surprenant de s'interroger sur le sens à donner à la notion de personne dans une philosophie comme celle de Spinoza, c'est que nous sommes accoutumés à envisager cette notion uniquement en terme de Substance. En conséquence un monisme comme celui de Spinoza qui ne conçoit l'homme que comme un mode de la substance ne pourrait d'aucune manière intégrer une telle notion.

Cependant si l'on y regarde d'un peu plus près, la philosophie de Spinoza, qui se présente avant tout comme une éthique, c'est-à-dire comme une pensée orientée dans une perspective pratique dont l'objectif est la réalisation de la perfection humaine, ne réduit pas l'homme à une chose parmi les choses.

Si l'homme est dans la nature un être comme les autres, il n'est pas pour autant un être quelconque, il a pour l'homme une valeur indiscutable, il représente même la plus haute valeur, dans la mesure où rien n'est plus utile à un homme qu'un autre homme, rien n'est plus utile à l'homme qu'un homme guidé par la raison.

En conséquence, si d'un point de vue ontologique il n'y a pas d'anthropologie spinoziste, l'homme n'étant pas « comme un État dans l'État », d'un point de vue éthique il peut sembler cohérent de considérer que l'homme n'est pas pour Spinoza sans dignité. Ce qui autorise à penser la présence dans sa philosophie d'une conception implicite de la personne.

\section{LA PERSONNE COMME PROBLÈME}

Pour la grande majorité d'entre nous, le caractère sacré de la personne humaine semble ne faire aucun doute, mais si l'on approfondit un tant soit peu

1. Mes remerciements à Jacqueline Lagrée pour ses conseils avisés qui m'ont permis de préciser et d'enrichir le contenu de ma réflexion. 
l'analyse de cette notion, des difficultés qui sont sources de divergences plutôt que de consensus se présentent.

À partir de quel stade de son développement un être humain devient-il une personne et quand cesse-t-il de l'être? L'est-il dès sa conception ou le devient-il au cours de son développement ${ }^{2}$

Même le droit qui donne l'impression de reposer sur des conventions fermes semble à ce sujet cultiver l'ambiguïté. Ainsi pour la loi française, l'être humain n'est une personne juridique qu'à compter de l'heure et du jour de sa naissance. Cependant la loi sur l'Interruption Volontaire de Grossesse impose un délai au-delà duquel un avortement n'est plus autorisé. Le statut du fœtus est alors problématique puisque ne bénéficiant pas du statut de personne, il n'est pas non plus considéré comme une simple chose, comme un vulgaire agrégat de cellules.

Ces difficultés nous conduisent à abonder dans le sens de ce qu'écrit Jacqueline Lagrée à ce sujet :

La question de la définition de la personne humaine, de ses délimitations (quand commence-t-elle et quand finit-elle?), de ses déterminations, de sa valeur et de sa dignité, est une question terriblement complexe et centrale en éthique tout court et plus particulièrement en éthique médicale. ${ }^{3}$

La philosophie de Spinoza n'est-elle pas en mesure de nous aider à résoudre cet ensemble de problèmes? Chez Spinoza la notion de personne n'est jamais mentionnée, et pourtant sa philosophie se présente comme une éthique. Est-ce à dire que Spinoza a élaboré une éthique qui serait susceptible de se passer de la notion de personne?

Ou bien, n'y a-t-il pas une conception implicite de la personne qui pourrait se dégager de la pensée de Spinoza? Le monisme fait, certes, de l'homme un être comme les autres dans la nature, il n'en fait pas pour autant un être quelconque, dans la mesure où il est apparemment le seul être qui puisse accéder à la liberté grâce à la connaissance.

\section{LA NOTION DE PERSONNE DANS LA TRADITION PHILOSOPHIQUE}

Traditionnellement, la notion de personne se fonde sur l'idée que l'homme est en quelque sorte une exception dans la nature, un être doté d'une dimension morale à laquelle les autres êtres ne pourraient pas accéder. Le plus souvent cette dimension repose sur la présence en l'homme d'un principe spirituel dont la nature lui serait spécifique et qui le différencierait des autres êtres vivants, l'âme distincte du corps, par exemple.

C'est cette distinction qui est présente dans la philosophie cartésienne qui conçoit l'homme comme un être duel constitué de l'union d'une âme (siège de la pensée) et d'un corps (relevant de la substance étendue), alors que les animaux, réduits à n'être que des corps, ne sont que des machines sans âme. C'est d'ailleurs pour cette raison que selon Descartes, l'homme occupe une place particulière dans la création.

\footnotetext{
2. On peut reprendre à ce sujet le questionnement formulé par Lucien Sève : « Peut-on considérer comme une personne un être en genèse qui jusqu'à sa troisième semaine ne présente pas même encore ce sillon neural à partir duquel se formera ce qui deviendra au septième mois seulement un système nerveux central vraiment fonctionnel? Peut-on tenir pour une personne ce qui jusqu'au quatorzième jour peut se diviser en deux jumeaux? Et que veut dire « dès l'instant de la conception », quand on sait que la pénétration du spermatozoïde dans l'ovocyte et l'union qui en résulte entre les chromosomes de ces deux gamètes est un processus durant toute une journée et marqué de phases? » Lucien Sève, Qu'est-ce que la personne humaine? p. 15, Éditions La Dispute, 2006.

3. Jacqueline Lagrée, Le médecin, le malade et le philosophe, chapitre 1, p. 21, Bayard Éditions, 2002.
} 
Cependant, bien que Spinoza ne partage pas cette position dualiste, il n'en accorde pas moins à la vie humaine une valeur propre et spécifique, ce qui nous conduit à penser qu'il y aurait, en un certain sens, une conception implicite de la notion de personne dans l'éthique spinoziste, conception qui pourrait peut-être nous aider à résoudre certains des problèmes auxquels se trouve confrontée la médecine contemporaine.

Avant de répondre à cette question nous allons tout d'abord tenter de préciser l'origine de la notion de personne. C'est principalement, comme le souligne Suzanne Rameix, avec le christianisme que la notion de personne verra le jour:

En effet, la théologie chrétienne introduit dans la philosophie la notion de personne, qui n'existait pas dans la pensée gréco-romaine. [...] On peut dire de façon rapide, mais exacte, que c'est la pensée chrétienne - préparée par la pensée judaïque - qui introduit en Occident, l'idée d'homme, au sens contemporain d'un sujet unique, singularisé, libre et conscient. ${ }^{4}$

Des penseurs chrétiens comme saint Augustin et Boèce sont à l'origine de la naissance de cette notion de personne qui demeure au cœur de la morale occidentale même si cette dernière a tendance à se laïciser.

Dans la mentalité antique la notion de personne, au sens que nous lui donnons aujourd'hui, est pratiquement absente. Les hommes appartiennent à un cosmos ordonné et hiérarchisé dans lequel chacun occupe la place qui lui est due. Ils sont aussi les membres d'une communauté, d'un peuple ou d'une cité tout aussi ordonnée et hiérarchisée, et ne conçoivent leur identité que dans le cadre de cette appartenance. Ainsi on est grec ou barbare et il est difficile pour un Grec de concevoir que la vie d'un barbare vaut autant que la sienne. Dans un tel contexte, des pratiques, comme l'esclavage, qui aujourd'hui nous révoltent, ne choquent en rien la conscience des anciens Grecs ou des Romains. L'idée que tout homme en vaut un autre est quasiment impensable dans une telle représentation du monde. Même des penseurs aussi profonds et subtils que Platon et Aristote qui, c'est le moins qu'on puisse dire, n'étaient pas indifférents aux questions d'éthique, ne ressentaient aucun scrupule à posséder et à utiliser des esclaves, ces « outils animés ${ }^{5}$ dont Aristote justifie d'ailleurs la condition dans le Livre I de La Politique ${ }^{6}$.

Certes, plus tardivement, les Romains vont contribuer à l'élaboration de la notion de personne mais celle-ci n'aura qu'une signification juridique et ne comprendra pas cette dimension de sujet moral, unique et irremplaçable auquel la pensée chrétienne a donné le jour. La personne juridique ne désigne qu'un sujet ayant des droits et des devoirs relativement à la Cité. Cela dit, la notion de personne juridique n'est pas totalement étrangère à une réflexion ontologique et morale dans la mesure où la question se pose aujourd'hui de savoir en fonction de quel critère un être peut être considéré comme une personne et donc jouir de certains droits. C'est par exemple le cas du fœtus ou de l'embryon, mais aussi du déficient mental, du vieillard sénile ou du comateux profond. Mais ce type de question ne semble pas s'être posé dans le droit romain qui n'a apparemment pas envisagé la possibilité de concevoir la notion de personne hors du cadre de la personnalité juridique.

\footnotetext{
4. Suzanne Rameix, Fondements philosophiques de l'éthique médicale, Ellipses, 1996, p. 27.

5. Aristote, Éthique à Nicomaque, Livre VIII, chapitre XIII, traduction J. Tricot.

6. «Ces considérations montrent clairement quelle est la nature de l'esclave et quelle est sa potentialité: celui qui par nature, ne s'appartient pas à lui-même, tout en étant un homme, mais est la chose d'un autre, tout homme qui malgré sa qualité d'homme, est une propriété n'étant rien d'autre qu'un instrument d'action et séparé du propriétaire. » Aristote, Politique, traduction J. Tricot.
} 
Le christianisme va donc rompre avec cette conception de l'homme (si tant est que l'on puisse parler d'une idée de l'homme dans la pensée antique), pour envisager chaque être humain comme une créature aimée de Dieu et qui est l'égal, à ses yeux, de tous les autres hommes.

Saint Augustin, entre autres, va exprimer d'une manière originale la naissance de la conception d'un sujet libre et responsable dans ses Confessions, l'un des premiers ouvrages rédigés à la première personne et dans lequel il examine sa conscience. S'interrogeant sur le mal qu'il a pu commettre, sur ses fautes et la fragilité de sa foi, il s'exprime en tant qu'individu particulier responsable de lui-même, de ses actes et de ses pensées. Augustin rédige dans ses Confessions l'histoire de sa conversion, il s'agit en quelque sorte d'une autobiographie spirituelle, rédigée à la gloire de Dieu et au cours de laquelle il nous décrit l'itinéraire qui l'a conduit vers le christianisme.

Dans la Cité de Dieu Augustin développe même une réflexion qui fait que certains ont pu voir en lui un précurseur de Descartes, dans la mesure où il exprime l'évidence du sujet pensant qui s'apparaît à lui-même comme indubitablement existant:

Puisque donc je suis, moi qui me trompe, comment me puis-je tromper à croire que je suis, vu qu'il est certain que je suis si je me trompe? Ainsi puisque je serais toujours moi qui serais trompé, quand il serait vrai que je me tromperais, il est indubitable que je ne me puis tromper lorsque je crois que je suis. ${ }^{7}$

La pensée de Saint Augustin semble donc être à l'origine du sujet moderne et de la notion de personne fondée sur la conscience, elle-même enracinée dans la mémoire qui garantit la permanence du moi, la raison et la liberté de l'individu responsable. Ce sont ces trois critères (conscience, raison, liberté) qui seront traditionnellement retenus pour définir la notion de personne.

Cependant si la notion de personne apparaît implicitement dans la pensée augustinienne, elle n'est pas explicitement théorisée comme elle le sera ensuite chez Boèce qui dans son Traité sur le Christ donne une définition de la personne comme:

substance individuelle de nature rationnelle ${ }^{8}$

S'interrogeant sur ce qui, parmi les créatures de Dieu, peut être qualifié de personne, les choses comme les êtres vivants, seuls finalement les hommes considérés chacun dans leur singularité peuvent être considérés comme des personnes:

Mais parmi toutes ces substances, nulle part la personne ne peut se dire dans les universelles, mais seulement dans les singulières et dans les individuelles. En effet, il n'y a aucune personne de l'animal ou de l'homme en général, mais c'est bien à Cicéron, à Platon, à des individus singuliers que l'on donne le nom de personne singulière. ${ }^{9}$

C'est donc à la fois par sa rationalité et dans sa singularité que chaque homme peut être considéré comme une personne.

Ainsi aux trois caractéristiques précédentes que sont la conscience, la raison et la liberté vient s'en ajouter une quatrième, la substantialité. Est considéré comme personne tout être qui peut être considéré comme subsistant par soi, comme étant le substrat des qualités énoncées précédemment et de ce qui fait la singularité de la personne elle-même.

Cette idée d'un substrat permanent, support de ses qualités essentielles mais aussi de toutes les modifications particulières que peut subir l'être humain au cours de son existence, cette idée de la personne comme l'invariant qui subsiste malgré

7. Augustin, La cité de Dieu, Livre XI, chapitre 24.

8. Boèce, Traité sur la personne et les deux natures du Christ. III.

9. Ibid, II. 
toutes les variations de la personnalité, ce qui fait donc l'identité d'un être quel que soit le moment de sa vie; cette idée se retrouvera également chez le philosophe anglais John Locke pour qui la personne est:

un être pensant et intelligent, capable de raison et de réflexion, et qui peut se considérer comme étant le même, comme étant la même chose pensante, en différents temps et en différents lieux. ${ }^{10}$

Cependant chez Boèce une catégorie supplémentaire intervient dans la définition de la personne, celle de relation. Il s'interroge sur la question de La Trinité et se demande comment Dieu peut être, pour les chrétiens, une personne à part entière, alors qu'il est constitué de ces trois personnes que sont le Père, le Fils et le SaintEsprit?

Mais puisque le Père est Dieu, que le Fils est Dieu et que l'Esprit Saint est Dieu, et que Dieu n'a aucune différence qui le rendrait différent de Dieu, c'est que Dieu ne diffère en rien d'aucune des personnes divines. Or, là où les différences sont absentes, la pluralité est aussi absente, et là où est absente la pluralité, là est présente l'unité. Rien d'autre n'a pu être engendré de Dieu que Dieu. Enfin, dans ce qui est numérable, la répétition des unités ne produit pas, en tous ses modes, la pluralité. ${ }^{11}$

Cette unité de Dieu dans La Trinité est donc maintenue parce que la personne se définit également comme relation avec un alter ego. Être une personne ce n'est pas en effet vivre isolé de ses semblables, c'est essentiellement tisser un réseau de relations avec eux, relations qui font être tant les autres que soi-même. Cette dimension se traduit dans la personne de Dieu par La Trinité qui permet de le penser à la fois comme substance et comme relation:

Ainsi donc, la substance maintient l'unité, la relation introduit un élément multiple dans La Trinité, et, de cette façon, (les personnes de La Trinité) sont énoncées, chacune en particulier et séparément, uniquement comme relevant de la relation. Car le Père n'est pas le même que le Fils, et l'un et l'autre ne sont pas les mêmes que l'Esprit Saint. Et pourtant, c'est le même Dieu qui est Père, Fils et Esprit Saint, le même qui est juste, le même qui est bon, le même qui est grand, le même qui est tout ce qu'il est possible de lui attribuer par soi. ${ }^{12}$

La personne peut donc se définir comme un être substantiel qui possède l'intuition de son existence et de celle d'autrui avec qui il est en relation, qui peut user de sa raison pour distinguer le vrai du faux et le bien du mal et qui dispose d'une liberté qui lui permet de choisir. Par cette définition la personne humaine acquiert une dignité morale qui va conduire un philosophe comme Kant à penser qu'elle constitue une fin en soi :

Agis de telle sorte que tu traites l'humanité aussi bien dans ta personne que dans la personne de tout autre, toujours en même temps comme une fin, et jamais simplement comme un moyen. ${ }^{13}$

Le respect de la personne humaine est donc au cœur même de la loi morale, de l'impératif catégorique qui me dicte de toujours agir par devoir, par respect pour la loi morale qui procède d'une raison pratique universelle. Cet impératif repose principalement sur l'idée que la liberté humaine résulte de la place particulière qu'occupe l'homme dans le monde, il serait le seul être à pouvoir s'affranchir des lois de la nature et à pouvoir se donner, grâce à sa raison, une loi valable pour tous et à laquelle il

10. John Locke, Essai philosophique concernant l'entendement humain, (1694), II, 27.

11. Boèce, De Trinitate, $\S 5$.

12. Ibid, §6.

13. Kant, Fondements de la métaphysique des mœurs, deuxième section, trad. V. Delbos (1907). 
obéirait librement par devoir. Ainsi selon Kant la personnalité consiste dans la capacité qu'a l'homme de s'élever au-dessus de la nature et au-dessus de lui-même:

Ce n'est autre chose que la personnalité, c'est-à-dire la liberté et l'indépendance vis-àvis du mécanisme de la nature entière, considérée en même temps comme la faculté d'un être qui est soumis à des lois particulières, à savoir aux lois pures pratiques données par sa propre raison. ${ }^{14}$

Reste à savoir comment il est possible pour l'homme de s'affranchir des lois de la nature, affranchissement qui tombe sous le coup de l'analyse que fait Spinoza de l'illusion de la liberté propre à ceux qui ont conscience de leurs volitions mais ignorent les causes qui les déterminent. Certes, l'action droite est action selon la raison, mais il ne s'agit en rien d'une raison pure pratique, il s'agit de la connaissance qu'a l'homme des lois qui régissent son union avec la nature et qui déterminent ses actions par l'amour qu'il en retire pour ses semblables, augmentant ainsi sa puissance d'agir et sa joie.

D'ailleurs, notre lien à la nature se trouve confirmé par l'état de l'enfant dont le corps n'est pas encore totalement achevé, ou par la maladie qui, en nous affectant, peut nous faire perdre nos facultés et par conséquent l'usage de notre raison ainsi que la conscience que nous avons d'exister. Ainsi le tout jeune enfant ne dispose-t-il pas encore des facultés qui selon les critères que nous venons d'évoquer font une personne, il en est de même pour le malade en situation de coma profond ou le vieillard atteint de la maladie d'Alzheimer. Doit-on pour autant ne pas reconnaître à ces êtres le statut de personne et ne pas les traiter avec humanité?

Faire reposer la dignité de la personne humaine sur une supposée dimension surnaturelle de l'homme semble donc poser problème lorsque c'est précisément la nature qui par son action, la maladie ou le vieillissement, efface toute manifestation de cette dimension.

Mais à l'inverse, peut-on encore penser la notion de personne si l'on conçoit l'homme comme une simple partie de la nature?

Comment donc concevoir la notion de personne selon une conception déterministe de la nature dans laquelle l'homme peut certes être considéré comme un être conscient et doué de raison, mais ne peut en aucune manière être défini comme un être substantiel disposant d'une liberté de l'ordre d'un libre arbitre?

La philosophie de Spinoza est peut-être en mesure d'apporter une solution à ce problème.

\section{LA CONCEPTION IMPLICITE DE LA PERSONNE CHEZ SPINOZA}

\section{Personne et individu}

S'il y a une conception spinoziste de la personne, elle ne peut, en tout cas, se fonder sur une individualité qui serait le propre de l'homme. En effet, dans la Nature tous les modes sont des individus, qu'ils soient vivants ou non vivants et parmi les vivants qu'ils soient humains ou non humains. L'homme n'est donc pas différent des autres choses sur ce plan, car comme tout mode, il est un corps composé d'autres corps et une âme qui n'est autre que l'idée de ce corps. En effet, contrairement à son étymologie, le terme d'individu ne désigne pas, chez Spinoza, ce qui est indivis et indivisible, mais ce qui est composé et comporte un principe interne d'unité.

Quand quelques corps, de la même grandeur ou de grandeur différente, subissent de la part des autres corps une pression qui les maintient appliqués les uns sur les autres

14. Kant, Critique de la raison pratique, Traduction par J. Gibelin, Vrin, Paris, 1974, P. 99. 
ou, s'ils se meuvent avec le même degré ou des degrés différents de vitesse, les fait se communiquer les uns aux autres leur mouvement suivant un certain rapport, nous disons que ces corps sont unis entre eux et que tous composent ensemble un même corps, c'est-à-dire un Individu qui se distingue des autres par le moyen de cette union du corps. ${ }^{15}$

Ce qui fait l'individualité d'un mode c'est donc son type de composition. Ses parties sont à ce point adaptées les unes aux autres qu'elles restent réunies et maintiennent ainsi la structure de l'individu qu'elles composent le plus longtemps qu'il est possible. Autrement dit, un individu n'est pas un être définitivement indivisible, mais un être composé qui s'efforce de maintenir sa composition le plus longtemps possible. Ainsi, même s'il perd certaines de ses parties, il ne perdra pour autant son individualité. Dans la mesure où il se définit, non seulement, par les rapports internes que ses parties entretiennent entre elles, mais aussi par les rapports externes qu'il entretient avec son environnement, il parvient par recomposition à renouveler les parties manquantes sans que pour autant ses rapports de composition s'en trouvent, quant à eux, affectés. En conséquence son individualité n'en sera pas modifiée.

Si d'un corps, c'est-à-dire d'un individu composé de plusieurs corps, on suppose que certains corps se séparent et qu'en même temps d'autres en nombre égal et de même nature occupent leur place, l'Individu retiendra sa nature telle qu'auparavant sans aucun changement dans sa forme. ${ }^{16}$

Les parties manquantes peuvent même n'être renouvelées que partiellement, ou au contraire la recomposition peut donner lieu à un excès relativement à l'état initial, mais tant que la quantité retranchée ou superflue n'affecte pas la structure de ce corps et n'entraîne pas sa désagrégation, ce dernier conserve son unité et reste un individu. Je puis cueillir des fruits sur un arbre, ces derniers ne seront pas immédiatement remplacés, mais cet arbre n'en perdra pas pour autant son individualité. De même mon poids peut varier de quelques grammes ou de quelques kilos selon la nature de mon alimentation, mon organisme n'en perd pas nécessairement son unité. Pour que l'unité et donc l'individualité d'un corps se trouve affectée, il faut dépasser un seuil quantitatif, qui d'ailleurs peut varier d'un individu à l'autre, mais il faut également que les éléments en défaut ou en excès modifient de manière décisive les rapports de composition qui unissent les constituants de ce corps.

Les individus se différencient donc les uns des autres en fonction de la quantité de leurs composants, de la diversité de ces derniers et de la complexité des rapports qui les rassemblent. L'homme est donc de ce point de vue un individu comme les autres. La question se pose donc de savoir ce qui fait sa spécificité. Sur quoi pourrait-on faire reposer la différence de l'homme avec les autres modes? Peut-être précisément sur la diversité des parties qui le composent et sur la complexité des rapports qui les unissent?

En effet, toutes les propriétés qui caractérisent les individus et qui sont énoncées dans les Axiomes et les Lemmes qui suivent la Proposition XIII de la seconde partie de l'Éthique valent pour tous les corps et ne concernent pas simplement l'homme. Ce qui concerne plus précisément l'homme sera défini dans les six Postulats qui suivront.

Ces postulats ne peuvent suffire à définir l'homme, mais exposent comment les lois exposées précédemment, et qui concernent, répétons-le, tous les corps, se manifestent de façon particulière chez l'homme. Ce qui apparaît à la lecture de ces postulats, 
c'est qu'ils insistent sur la complexité de l'individu humain. Ainsi le premier d'entre eux insiste sur le degré de composition du corps humain:

Le corps humain est composé d'un très grand nombre d'individus (de diverses natures) dont chacun est très composé.

Le troisième postulat insiste également sur la richesse des relations que l'homme entretient avec le monde extérieur:

Les individus composant le Corps humain sont affectés, et conséquemment le Corps humain lui-même est affecté, d'un très grand nombre de manières par les corps extérieurs.

Sans aller jusqu'à commenter la totalité de ces six postulats, nous pouvons conclure, si nous nous limitons à ceux que nous venons de citer, que la spécificité de l'homme réside, non seulement dans le nombre, mais aussi et surtout dans la diversité des parties qui le composent (il est composé d'un grand nombre de parties de diverses natures), ainsi que dans la complexité et la diversité des rapports qui l'unissent à la totalité dont il fait partie (il est affecté, d'un très grand nombre de manières par les corps extérieurs). Autrement dit, cette spécificité repose essentiellement sur des facteurs initialement quantitatifs. La question se pose donc de savoir si cette différence quantitative peut ensuite donner lieu à un saut qualitatif qui pourrait nous conduire à penser tout homme comme une personne.

\section{Une conception relationnelle de la personne}

L'usage que fait Spinoza du terme de personne dans son œuvre autorise à penser que cette notion n'est pas chez lui théorisée au point de jouer un rôle opératoire et fondamental dans sa pensée ${ }^{17}$. Cependant dans la mesure où il s'efforce de préciser ce

17. On trouve en effet sous la plume de Spinoza le terme de persona dans certaines expressions dans lesquelles il désigne des hommes en disant « une personne » ou « des personnes », il utilise également ce terme dans son sens grammatical lorsqu'il s'interroge dans le Traité théologico-politique afin de savoir si une parole a été prononcée en "première personne ».

Ainsi, par exemple, dans le chapitre premier du Traité théologico-Politique (p. 34, 35) lorsqu'il s'interroge sur la nature de la voix qui a transmis le Décalogue aux Israélites, Spinoza écrit: "Car il semble bien contraire à la raison d'admettre qu'une chose créée, dépendant de Dieu en la même manière que les autres, puisse en son propre nom exprimer ou expliquer l'essence ou l'existence de Dieu matériellement ou en parole; à savoir en disant à la première personne: je suis Jehova votre Dieu. (in prima persona, ego sum Jehova Deus tuus, etc.) 》 Spinoza emploie aussi le terme de personne dans un registre plus politique lorsqu'il distingue dans le $\S 2 \mathrm{du}$ chapitre X du Traité Politique entre la personne naturelle et la personne civile: "C'est pour satisfaire à ces conditions que nous avons subordonné à l'assemblée générale un conseil des syndics, de façon que le glaive perpétuel ne soit pas au pouvoir d'une personne naturelle, mais d'une personne civile dont les membres sont trop nombreux pour qu'ils puissent se partager l'État ou s'accorder pour un crime... (Ut igitur has omnes conditiones obtineamus, Syndicorum Concilium Concilio supremo subordinandum diximus, ut scilicet dictatorius ille gladius perpetuus esset non penes personam aliquam naturalem, sed civilem, cujus membra plura sint, quam ut imperium inter se possint dividere (per Art. 1. et 2. Cap. 8. (46), vel in scelere aliquo convenire: ad quod accedit, quod a reliquis imperii muneribus subeundis prohibeantur, quod militiae stipendia non solvant, et quod denique ejus aetatis sint, ut praesentia, ac tuta, quam nova, et periculosa malint.) » (p. 106, 107).

On trouve de plus le terme de «personalitas » sous la plume de Spinoza dans les Pensées Métaphysiques lorsqu'il s'interroge au sujet de ce qui pourrait distinguer l'essence, l'entendement et la volonté de Dieu: «nous n'ignorons pas le mot (celui de personnalité [personalitas scilicet]) qu'emploient à l'occasion les philosophes, pour expliquer la chose; mais si nous connaissons le mot nous en ignorons la signification et nous n'en pouvons former aucun concept clair et distinct; [...]» (p. 372, 373).

En revanche nous n'avons pas trouvé mention dans les textes de Spinoza de la personne humaine en tant que telle, Spinoza écrira « une personne » ou « des personnes » pour désigner un individu ou un ensemble d'individus particuliers, il aura recours à la notion de personne dans son sens grammatical (en première, deuxième ou troisième personne), il utilisera le terme de personne pour désigner un statut juridique ou politique, mais jamais, apparemment, il ne donnera à ce terme de personne la signification morale que nous lui donnons aujourd'hui. 
qui fait la spécificité de l'homme dans la nature, il est permis de s'interroger sur la présence d'une conception implicite de la notion de personne dans sa pensée.

En effet, si pour Spinoza l'homme n'est pas dans la Nature « comme un État dans l'État », il n'en est pas moins un être singulier dont la nature fait qu'il ne peut ni se considérer ni être considéré en tant que simple chose ou même tel un animal.

Comme le fait remarquer Robert Misrahi, la philosophie de Spinoza, parce qu'elle est une éthique, ne se réduit pas à un pur système métaphysique, elle est aussi une théorie de l'homme:

La doctrine de Spinoza est une philosophie parce qu'elle est un système du monde, rationnel et démonstratif, mais cette philosophie ne se réduit pas (comme on l'a cru trop longtemps) au système du Dieu-Nature: la théorie de la nature n'est qu'une condition préliminaire à l'établissement d'une théorie de l'homme, et celle-ci est destinée à fonder une éthique. Cette éthique est un chemin de vie, une «voie » qui conduit à la liberté et à la sérénité. ${ }^{18}$

D'ailleurs, Spinoza lui-même, dans le Traité politique, définit ainsi ce qu'il entend par la notion de vie humaine:

[...], celle qui ne se définit pas uniquement par la circulation du sang et par les autres fonctions communes à tous les animaux mais essentiellement par la raison et par la vertu et la vie véritable de l'esprit. ${ }^{19}$

Cela se trouve d'ailleurs confirmé par les premières pages du Traité de la réforme de l'entendement dans lesquelles Spinoza, s'interrogeant sur les biens qui méritent d'être recherchés, s'efforce de définir ce qu'il faut poursuivre pour mener une vie authentiquement humaine, c'est-à-dire une vie dans laquelle s'exprime pleinement ce qui fait la singularité de la nature de l'homme.

Ce texte qui pourrait apparaître comme le récit d'une conversion s'en distingue par le fait qu'il ne s'agit pas de la réponse à un appel qui viendrait de l'extérieur ou qui serait déterminé par un idéal de vie prédéfini. Pierre François Moreau souligne d'ailleurs ce point dans son commentaire du prologue au Traité de la réforme de l'Entendement :

Quelles que soient les similitudes, il y a cependant une différence majeure - non pas la différence externe qui tient à ce qu'il ne s'agit pas d'une conversion à l'orthodoxie, mais une différence interne et elle est décisive. Cette conversion est de part en part naturelle; elle ne connaît pas d'appel extérieur, pas d'autorité supérieure et, si elle s'oriente vers un Souverain Bien dont on apprendra ailleurs dans le système qu'il consiste dans la connaissance de Dieu, il faut noter que le mot Dieu, justement, n'est jamais prononcé dans ces pages; ce qui est après tout remarquable sous la plume d'un philosophe dont l'œuvre principale intitulera sa première partie: De Dieu. ${ }^{20}$

Il s'agit donc plus exactement d'une réorientation de la vie issue d'une insatisfaction relative à la vie ordinaire; d'une réorientation dont le principe actif est totalement immanent à la réflexion, puisque rien d'extérieur à celle-ci ne vient proposer de réponses aux questions qui résultent de la déception initiale vis-à-vis de ces biens ordinaires que sont les richesses, les honneurs et le plaisir. Ce point nous semble important à souligner car cette puissance immanente à la réflexion, qui lui fait trouver par elle-même, au fur et à mesure de sa progression, les réponses aux questions qu'elle produit, pourra peut-être servir de modèle pour aider certains malades à penser autrement leur maladie afin de la vivre autrement et de se soigner différemment.

18. Robert Misrahi, Spinoza, Éditions Médicis-Entrelacs, Paris, 2005, p. 20.

19. Traité politique, V, 5 ; cité par Jacqueline Lagrée, Le médecin, le malade et le philosophe, chapitre 1, p. 22.

20. Pierre François Moreau, Spinoza - L'expérience et l'éternité, Chapitre Premier, p. 34. 
On pourrait donc voir, dans ce parcours décrit par Spinoza au début du Traité de la Réforme de l'Entendement, l'expression d'une crise existentielle, à l'origine de l'orientation vers cette « vie nouvelle » qui est présentée comme la réalisation de la perfection humaine. Mais à la condition de ne pas confondre ce que nous expose le narrateur avec un récit qui se réduirait à une simple autobiographie intellectuelle propre à l'auteur de ce texte. Comme l'explique Pierre François Moreau, l'expérience à laquelle va se référer le narrateur du Traité concerne finalement tous les hommes qui sont en mesure de juger des limites relatives à la recherche des biens ordinaires:

[...] l'expérience, c'est ce terrain commun à toutes les vies individuelles, qui est d'une certaine façon enregistrée dans la mémoire de tous les individus, bien qu'elle ait été pour chacun remplie d'une façon diverse; l'un a été marchand dans la communauté juive d'Amsterdam, l'autre luthérien, médecin et directeur de théâtre, le troisième noble allemand et mathématicien... mais tous ont vu, fréquenté, ou subi, les honneurs, le plaisir et l'argent. ${ }^{21}$

Spinoza exprime cette insatisfaction à l'égard des biens de la vie commune dès les premières lignes de ce texte pour énoncer ensuite en quoi consiste l'objet de sa recherche:

L'expérience m'avait appris que toutes les occurrences les plus fréquentes de la vie ordinaire sont vaines et futiles; je voyais qu'aucune des choses, qui étaient pour moi cause ou objet de crainte, ne contient rien en soi de bon ni de mauvais, si ce n'est à proportion du mouvement qu'elle excite dans l'âme: je résolus enfin de chercher s'il existait quelque objet qui fût un bien véritable, capable de se communiquer, et par quoi l'âme, renonçant à tout autre, pût être affectée uniquement, un bien dont la découverte et la possession eussent pour fruit une éternité de joie continue et souveraine. ${ }^{22}$

Si les termes d'expérience et de vie sont ici à retenir comme fondamentaux, c'est que leur emploi permet à Spinoza de signifier que pour lui la vie humaine ne peut se réduire à son sens biologique, ni même se résumer à une vie sociale superficielle faite d'apparences et de faux-semblants. En effet, les termes d'expérience et de vie renvoient ici à ce que l'on désignerait aujourd'hui par le terme de « vécu », désignant précisément ce que ressent le sujet conscient dans sa relation au monde qui l'entoure.

L'expérience se donne donc à lire selon trois registres: ce que j'ai vécu; ce que j'ai vu les autres vivre; ce que nous avons appris ensemble que d'autres ont vécu et nous ont transmis, et que répète ce que nous vivons. ${ }^{23}$

Or, le narrateur du Traité semble ici ressentir son rapport au monde en termes de déchirement, rien ne lui convient de tout ce qui est communément présenté comme devant être désiré. Tout cela lui apparaît vain et futile, autrement dit vide, sans consistance et sans intérêt. Cette expérience de la vie va permettre que se révèle à la conscience ce qui fait la spécificité de l'homme dans la nature, ce qui fait qu'il ne peut simplement se contenter de subsister, de survivre comme n'importe quel être vivant. Il ressent parce qu'il est un être pensant des désirs d'une tout autre nature, et ce sont ces désirs qui font de lui une personne, un être qui, pour lui-même et pour tous ses semblables, vaut plus que tous les autres. Et, en effet, la complexion de l'homme est telle qu'il constitue un mode d'être qui a ses qualités propres, la conscience et la raison qui font de lui un être pensant ${ }^{24}$ ainsi qu'un être social dans la

21. Ibid. p. 57.

22. Traité de la réforme de l'entendement, § 1.

23. Pierre François Moreau, Spinoza - L'expérience et l'éternité, Chapitre Premier, p. 59.

24. «L'homme pense », Éthique, Deuxième partie, Axiome II. 
mesure où il a besoin de ses semblables pour progresser vers la perfection ${ }^{25}$. En ce sens la dimension morale de l'homme est incontestable, et c'est d'ailleurs ce qui explique que la philosophie de Spinoza est aussi une éthique, malgré le déterminisme foncier qui la caractérise. Certes, l'homme ne peut être pensé dans un tel contexte comme un être substantiel, il n'empêche que, comme le souligne Henri Atlan, sa dimension de sujet n'est pas pour autant totalement évacuée dans la mesure où sa complexion lui permet de disposer d'une pensée réflexive et d'un langage grâce auquel cette dernière peut être objectivée :

Le sujet humain spinoziste, évidemment très différent d'un sujet transcendantal, n'en existe pas moins comme un individu dont le corps est suffisamment complexe pour que l'idée de ce corps soit douée de réflexivité langagière. Chaque être humain en fait l'expérience, ne serait-ce que d'une façon inadéquate parce que limitée, dans sa propre singularité, et même parfois de façon adéquate à travers les notions communes de la raison. ${ }^{26}$

Le refus de penser l'homme comme un être substantiel (il ne peut en effet y avoir d'autre substance que Dieu ou la Nature) et par conséquent (puisqu'il ne peut plus être pensé que comme une partie de la Nature soumise au déterminisme) le refus de le penser comme un être disposant d'un libre arbitre, ne s'opposent pas à la possibilité de considérer l'homme comme une personne. Peut-être même ces deux refus sont-ils à l'origine de la possibilité de penser la notion de personne dans un contexte spinoziste?

C'est en effet par la puissance réflexive de sa pensée qui lui permet de se regarder et, en un certain sens, de s'objectiver, que l'être humain se constitue comme personne. Ainsi malgré son statut relatif au reste de la nature, l'homme est en mesure de se construire comme une unité susceptible de perdurer quels que soient les changements qui peuvent l'affecter. On pourrait reprendre ici, sans pour autant faire d'amalgame, l'expression qui sert de titre à un ouvrage de Paul Ricœur; on pourrait dire que c'est en effet la capacité qu'a l'homme de se penser comme un autre ${ }^{27}$ qui lui permet de construire son identité de personne, de se constituer comme un «soi » réfléchi et pas seulement comme un «je » immédiat. Finalement le sujet ne disparaît pas derrière toutes les déterminations qui le structurent, ce sont au contraire ces déterminations, quand elles sont ressaisies par la pensée, qui le constituent comme sujet et lui donnent sa dignité de personne. Dignité qui résulte, non seulement de la saisie de l'individu humain par lui-même, mais aussi de sa perception par ses semblables. C'est pourquoi, même lorsqu'il n'est pas encore un sujet (le nourrisson) ou qu'il ne l'est plus (le vieillard sénile ou le comateux profond), il reste cependant une personne dans la mesure où il est un semblable autre pour autrui.

L'homme, comme tout mode de la substance, est relié au reste de la Nature. Mais ce qui fait sa spécificité, c'est que, à la différence des autres modes, il est capable de penser. Il peut donc prendre conscience des liens qui l'unissent aux autres choses et s'efforcer de les tisser de la manière la plus adéquate qui soit. Certes, le sujet n'a pas d'identité substantielle, il n'est pas un noyau autour duquel se greffent ces liens, il est au contraire constitué par eux, il est le point de rencontre d'une multitude de connexions causales diverses (psychologiques, sociales, culturelles, économiques, biologiques). Par ces liens se constitue une identité qui relève plus, pour reprendre à nouveau un vocabu-

25. «Rien donc de plus utile à l'homme que l'homme; [...] », Ibid, Quatrième partie, scolie de la proposition XVIII.

26. Henri Atlan, Les étincelles de hasard, chapitre IV, note 84, p. 261.

27. Paul Ricœur, Soi-même comme un autre, Seuil, 1990. 
laire emprunté à Paul Ricœur, de l'ipséité (un soi qui change et diffère tout au long de sa vie) que de la mêmeté (qui renverrait à un noyau permanent de la personnalité). Toujours selon Ricœur, le soi découvre et construit son identité par le récit qu'il peut faire de sa vie. N'est-ce pas finalement ce que fait le narrateur des premières pages du Traité de la réforme de l'entendement, lorsqu'il raconte comment s'effectue la prise de conscience du caractère futile des biens ordinaires et comment s'ouvre la voie vers la perfection humaine. Ne pourrait-on pas voir dans cet aspect de l'œuvre de Spinoza une dimension narrative du cheminement éthique? C'est donc à partir de ce récit que nous allons tenter de mettre en évidence la manière dont se constitue implicitement une certaine conception de la personne dans la philosophie de Spinoza.

\section{La dimension narrative et réflexive de la personne}

Dans le courant de sa réflexion, concernant les maux auxquels s'exposent communément les hommes qui se laissent emporter par des désirs immodérés et irréfléchis, le narrateur du prologue du Traité écrit:

Ces maux d'ailleurs semblaient provenir de ce que toute notre félicité et notre misère ne résident qu'en un seul point: à quelle sorte d'objets sommes-nous attachés par l'amour? ${ }^{28}$

Quelle est donc cette vie nouvelle à laquelle il aspire, sinon une vie habitée par un amour se portant sur une « chose éternelle et infinie » et qui « repaît l'âme d'une joie pure, d'une joie exempte de toute tristesse »? L'objet de cet amour est un «bien grandement désirable » et mérite « qu'on le cherche de toutes ses forces » ${ }^{29}$. Par cet amour l'homme peut accéder à une « nature supérieure » qui consiste en « la connaissance de l'union qu'a l'âme pensante avec la nature entière. ». Le souverain bien ne peut simplement consister ici à vivre en accord avec la nature, nous vivons de fait en accord avec la nature. Il est par définition impossible de se désolidariser des lois de la nature et de s'opposer à elles. Le souverain bien résulte plus exactement de la connaissance de cet accord, de la connaissance des lois qui nous unissent à la nature. Cette connaissance n'étant pas sans effet, elle nous transforme et nous permet de vivre cet accord consciemment, activement et d'une manière qui nous convienne. Nous pouvons ainsi conquérir une liberté qui consiste dans la capacité d'agir selon la seule nécessité de notre nature.

D'autre part cette nature supérieure ne s'acquiert pas dans la solitude. L'homme est aussi un être qui est conduit à vivre avec ses semblables et à s'accorder avec eux, s'il veut vivre en paix et en sécurité et progresser avec eux vers la perfection. Il est donc nécessaire que cette vie nouvelle puisse être partagée avec et par le plus grand nombre:

Telle est donc la fin à laquelle je tends: acquérir cette nature supérieure et faire de mon mieux pour que beaucoup l'acquièrent avec moi; car c'est encore une partie de ma félicité de travailler à ce que beaucoup connaissent clairement ce qui est clair pour moi, de façon que leur entendement et leur désir s'accordent pleinement avec mon propre entendement et mon propre désir. Pour parvenir à cette fin il est nécessaire d'avoir de la Nature une connaissance telle qu'elle suffise à l'acquisition de cette nature supérieure; en second lieu, de former une société telle qu'il est à désirer pour que le plus d'hommes possible arrivent au but aussi facilement et sûrement qu'il se pourra. ${ }^{30}$

28. Traité de la réforme de l'entendement, § 3.

29. Ibid.

30. Ibid, § 5 . 
C'est donc par la connaissance de la nature même des liens qui l'unissent à une Nature dont il est partie intégrante et par les relations qu'il entretient avec les autres hommes à l'intérieur de celle-ci, que l'homme peut conquérir sa dignité de personne.

C'est donc bien le caractère relatif de l'existence humaine qui est la condition pour que l'homme puisse accéder au statut de personne.

Ainsi, bien que n'ayant aucune réalité substantielle et ne disposant d'aucun libre arbitre, l'homme est-il en mesure de conquérir sa liberté, son statut et sa dignité de personne grâce à la réflexion qui lui permet de connaître son union à la Nature en s'interrogeant principalement sur les causes qui le déterminent. Autrement dit si la définition de la personne qui peut se dégager de la philosophie de Spinoza ne peut en aucun cas être substantialiste, elle s'accorde cependant avec la dimension relationnelle que nous avions soulignée chez un penseur comme Boèce. C'est par sa capacité à entretenir des relations avec ses semblables et avec la nature tout entière que l'homme peut être considéré comme une personne.

Grâce à ces relations les hommes entrent dans un processus de reconnaissance mutuelle par le moyen du langage qui leur permet à la fois de se représenter à euxmêmes ce qu'ils sont, mais aussi de rentrer en contact pour faire d'autrui un « interlocuteur possible ». C'est par cette expression qu'Edmond Ortigues caractérise la personne dans un article qui porte sur le concept de personnalité:

Qu'est-ce qu'une personne? Une personne est un être doué de raison ou de langage. Il en sera donc de la personnalité comme de la culture et du langage. Une culture n'est pas un système clos, puisque la culture est tout ce qui peut s'apprendre. De même, il n'existe que des langues particulières, pourtant chaque langue se propose comme un instrument universel de la pensée. De même encore, la personnalité n'existe que sous des caractéristiques morales particulières mais elle se définit universellement par la capacité de se reconnaître et de se faire reconnaître en communiquant avec d'autres par le moyen de langages et de symboles. La conscience de soi chez l'enfant se développe en même temps que la capacité de communiquer avec autrui. La singularité personnelle d'un individu se donne à reconnaître dans la question : comment puis-je communiquer avec lui ? La personne, c'est l'interlocuteur possible. Les êtres humains se reconnaissent comme des personnes dans la mesure où ils deviennent les uns pour les autres des interlocuteurs possibles. ${ }^{31}$

C'est donc tout d'abord la société qui fait de l'homme une personne, c'est par son intégration progressive dans une intersubjectivité constituée par la communication qui entraîne une reconnaissance réciproque, que le sujet humain accède au statut de personne. Mais c'est aussi par la connaissance, qui est avant tout connaissance de ce qui nous relie au monde extérieur, que nous évoluons en tant que personne.

En conséquence, par la pensée, l'homme, qui s'est éveillé à la philosophie et qui tend vers la sagesse, cherche à mieux comprendre les déterminations par lesquelles il est relié à Dieu ou à la Nature pour progresser vers une autonomie source de joie. Et, plus cette compréhension et cette connaissance s'accroissent, plus nous nous affirmons comme des sujets libres et autonomes, plus nous devenons des personnes.

Nous pouvons donc reprendre ici la formule utilisée par Jacqueline Lagrée:

On ne naît pas « personne », on le devient ${ }^{32}$.

31. Edmond Ortigues, «Le concept de personnalité », in Critique, n 456, 1985, p. 522-523.

32. Jacqueline Lagrée, Le médecin, le malade et le philosophe, chapitre I, p. 24. 
Et c'est précisément par les liens qui nous unissent au monde que nous devenons des personnes. Ces liens sont, d'une part, ceux qui nous relient à la nature, principalement sur le plan biologique, et d'autre part, ceux qui, à l'intérieur de cette nature, réunissent les hommes entre eux sur le plan social et relationnel ainsi que sur le plan symbolique et culturel:

La personne humaine ne se comprend donc que dans l'articulation de trois plans distincts mais liés: le plan biologique de l'individu, de son programme génétique et de ses montages innés; le plan relationnel de son être avec autrui, de son affectivité et de son histoire; le plan symbolique de l'inscription dans une culture, dans une langue, dans une tradition et de la reprise de sa vie dans un discours. ${ }^{33}$

Cette conception nous semble pouvoir s'accorder avec la détermination implicite de la personne qui se dégage de la pensée de Spinoza. Puisque cette philosophie pense l'homme comme un être relié au reste de la Nature et qui ne peut s'épanouir que dans l'union avec ses semblables à l'intérieur d'une société, il est donc en mesure de conquérir sa dignité par une connaissance de plus en plus précise et de plus en plus profonde des liens qui l'unissent à la totalité dont il fait partie et par le partage de cette connaissance avec les autres hommes.

\section{Le rôle de la connaissance dans la construction de la personne}

On pourrait certes opposer à cette conception de la personne qu'elle est très limitative, puisqu'apparemment, elle ne permet d'accorder le statut de personne qu'à celui qui a atteint un certain degré de connaissance et de réflexion. Est-ce à dire que l'ignorant n'est pas une personne? Ce serait aller un peu vite en besogne que d'en arriver à une telle conclusion. Si tel était le cas, nous n'aurions aucun égard pour l'ignorant et donc aucune raison de souhaiter qu'il progresse lui aussi vers la connaissance. Si tel était le cas, le sage ou en tout cas le philosophe, celui qui s'oriente sur la voie de la sagesse, ne chercherait pas à entraîner les autres hommes avec lui et ne chercherait pas à partager cette vie nouvelle à laquelle il aspire avec eux.

Si donc nous avons le désir de sortir de notre ignorance et de faire sortir l'ignorant de la sienne, c'est précisément que nous considérons ce dernier comme une personne. Autrement dit, le statut de personne se conçoit plus ici en terme de puissance de connaître, que d'actualité de la connaissance. C'est d'ailleurs la raison pour laquelle aucun homme n'est jamais totalement ignorant, car même le premier mode de perception est une connaissance. Il s'agit certes d'une connaissance qui doit être épurée, réformée, mais elle n'en contient pas moins des éléments à partir desquels l'homme peut progresser vers la béatitude.

C'est donc parce que la nature même de l'âme humaine est de connaître que tout homme est une personne, une personne en devenir certes, mais une personne néanmoins. Intrinsèquement le désir de connaître constitue la nature même de l'esprit, nous possédons tous au moins une idée vraie, et nos erreurs ne sont que des conséquences de facteurs extérieurs de diverses natures qui viennent faire obstacle à ce désir sans que nous nous en rendions compte. Si cette orientation naturelle de l'esprit humain vers la connaissance fait de l'homme une personne, c'est qu'elle lui permet également de progresser vers la liberté. Certes, cette liberté n'est pas de l'ordre du libre arbitre, il s'agit d'une liberté d'une tout autre nature qui peut se définir comme une adhésion sans contrainte à la nécessité. L’homme libre est celui qui, sans

33. Ibid, p. 25. 
y être conduit par aucune cause extérieure, épouse en suivant un mouvement intérieur la réalité dont il perçoit, grâce à sa connaissance, la perfection. Henri Atlan insiste d'ailleurs sur cette dimension de la connaissance pour exposer en quoi il n'y a pas nécessairement d'incompatibilité entre déterminisme et liberté :

Loin d'être opposé à l'idée de la liberté, c'est au contraire seulement le point de vue de cette connaissance infinie qui nous permet d'en concevoir la nature et la réalité. C'est seulement ce passage à la limite, conduisant à une connaissance potentiellement infinie du déterminisme absolu de tout ce qui est, qui nous permet de concevoir aussi la possibilité d'une liberté absolue où notre être serait confondu avec notre savoir. Contrairement à l'opposition classique entre libre arbitre et déterminismes naturels, où plus de science semble impliquer moins d'humanité, l'expérience même d'un déterminisme absolu et intemporel nous permet de concevoir, par l'extrapolation à l'infini vers ce que serait une connaissance totale de ce déterminisme, la possibilité d'une liberté d'une autre nature, différente du libre arbitre, où toujours plus de science voudrait dire toujours plus de liberté. ${ }^{34}$

En conquérant ainsi sa liberté l'homme devient une personne en tant que sujet éthique dans la mesure où il comprend les liens qui l'unissent aux autres hommes et à la nature tout entière. C'est en ce sens que ce progrès vers la connaissance constitue un appel à l'éthique :

Contrairement à l'idée reçue qui confond liberté et libre arbitre, nous voyons donc que plus de connaissance des mécanismes de nos comportements et de leurs déterminismes peut nous conduire à toujours plus de liberté, dans un va-et-vient entre l'expérience de notre finitude et l'intuition de l'infini, entre notre existence dans le temps et notre connaissance de l'intemporel. C'est là, entre l'infini que nous concevons et la finitude que nous atteignons, que se logent les exigences ou les appels à l'éthique.

Loin de disparaître, comme on le croit du fait de l'opposition supposée entre déterminisme et liberté, l'éthique y grandit, si l'on peut dire, au sens qu'elle devient adulte. C'est celle d'une responsabilité locale, subjective et limitée dans l'existence, mais potentiellement infinie dans son principe, parce qu'associée à la recherche infinie de la connaissance et de la pratique des lois humaines et non humaines, qui conduisent le monde. Cette « responsabilité limitée », si l'on peut dire, dans l'existence donnée, augmente au fur et à mesure des progrès de cette connaissance. ${ }^{35}$

C'est donc, à la fois, la dimension relationnelle de l'existence humaine et la tendance naturelle de l'esprit humain à connaître et comprendre les relations qui l'unissent à la nature et à ses semblables, pour conquérir sa liberté, qui font de l'homme une personne. Et cela vaut aussi pour le déficient mental, le vieillard ou même le comateux profond: s'ils sont encore des personnes, c'est qu'intrinsèquement leur nature est toujours celle d'un être désireux de connaître, mais dont le désir est entravé, voire écrasé, par une nécessité extrinsèque. De plus, ils demeurent des personnes en raison des relations que leurs proches continuent d'entretenir avec eux. Par l'amour et le respect qui leur sont adressés, ils ne sont pas des corps sans âme. S'ils ne sont pas ou plus des personnes pour eux-mêmes, ils sont et restent des êtres dignes de l'humanité pour ceux qui les entourent et prennent soin d'eux. Tout homme peut donc en conséquence accéder à la dignité de personne.

Peut-être en va-t-il autrement de l'embryon et du fœtus? N'ayant pas encore atteint un niveau de complexion suffisant pour développer une telle puissance, ils ne peuvent être considérés comme des personnes pleines et entières, mais plutôt, pour reprendre une expression utilisée dans plusieurs avis du CCNE, comme des «personnes potentielles ». Lucien Sève précise d'ailleurs à ce sujet, pour éviter tout glisse- 
ment vers une position essentialiste, avec laquelle les biologistes risqueraient d'être en désaccord, que l'embryon n'est pas une personne potentielle, mais doit être traité comme tel. Il distingue donc le concept de «personne potentielle » et la notion de «potentialité de personne »:

Plusieurs dossiers que le Comité (CCNE) eut à étudier ensuite portaient sur les procréations médicalement assistées. Et à ce sujet, nous trouvions dans la corbeille léguée par nos devanciers de l'INSERM une formule faisant autorité qu'avaient élaborée au cours de leurs discrètes rencontres le ministre de la recherche Hubert Curien et le cardinal Lustiger: «L'embryon humain est une personne potentielle. » Dans ses tout premiers avis, en 1983-1984, le CCNE a conservé cette formulation. Mais lorsque la tâche m'échut d'animer un groupe de travail sur la personne, il apparut sans tarder que la plupart des biologistes et médecins qui y participaient, y compris des croyants, n'admettaient absolument pas une telle formule. Affirmer que l'embryon est une personne potentielle ou non, n'a aucun sens, disaient-ils avec force, pour la dirimante raison que la personne n'est en rien une notion biologique. [...] Or à y réfléchir attentivement il apparaissait que la difficulté éthique renvoyait à une confusion philosophique. Aussi ai-je suggéré à mes collègues de distinguer en toute clarté deux notions d'apparence voisine mais d'essence complètement hétérogène: la personne potentielle en tant que concept purement et expressément éthique, et la potentialité de personne, ou pour être plus exact d'être humain, en tant que concept purement et expressément biologique. Nous dirons donc non pas du tout que "l'embryon est une personne potentielle ", énoncé confusionnel où le verbe être chosifie inacceptablement la valeur, mais qu'il « recèle une potentialité d'être humain », assertion biomédicalement évidente à laquelle personne n'objecte. Et c'est au nom de ce fait biologique incontestable que nous validons la démarche éthique consistant à le traiter comme une personne potentielle, c'est-à-dire à assigner des bornes à nos pouvoirs sur lui, en tant que cette simple potentialité nous enjoint de voir déjà en lui, dans une mesure à préciser, le sociétaire futur de l'ordre de la personne. ${ }^{36}$

Cette potentialité s'actualise dès la naissance du point de vue du droit, mais peut advenir plus précocement d'un point de vue moral et affectif. Le statut de l'embryon ou du fœtus qui répond à un projet parental peut déjà être apparenté à celui d'une personne dans la mesure où une relation singulière s'établit entre l'enfant à naître et ses parents.

\section{CONCLUSION}

Il reste maintenant à définir dans quelle mesure il est permis de parler de dignité à propos de la conception implicite de la personne qui se dégage de la pensée de Spinoza.

La notion de dignité renvoie initialement à la notion de distinction. Elle désigne dans la société aristocratique ce qui distingue celui qui fait partie de l'élite du reste de la société. L'évolution de ce terme a conduit à lui donner un sens plus universel, voire plus universaliste, la dignité concerne désormais tous les hommes et désigne ce qui fait la valeur spécifique de l'être humain. Ainsi, porter atteinte à la dignité humaine, c'est finalement traiter l'homme comme s'il ne se distinguait pas des autres êtres, le traiter comme une chose ou comme un vulgaire animal.

L'homme est donc détenteur d'une certaine dignité, parce que, pour lui-même, il vaut plus que les autres êtres qui constituent la nature. Parce qu'il accède par sa complexion à un mode d'existence qui lui est propre et qu'il partage avec ses sem-

36. Lucien Sève, Qu'est-ce que la personne humaine? La Dispute, 2006, p. 66-67. 
blables. Il ne peut être traité sans certains égards, sans un certain respect, c'est-à-dire sans ressentir envers lui-même et ses semblables le sentiment que lui inspire la reconnaissance de la valeur spécifique qui résulte de ce mode d'existence.

De ce point de vue, si l'on définit la dignité humaine comme ce qui fait la valeur distinctive de l'homme pour l'homme, par rapport à toutes les autres parties de la Nature, il est possible de parler de dignité humaine dans le cadre de l'éthique spinoziste. Certes, il ne s'agit pas d'une valeur absolue, il ne s'agit que de la valeur de l'homme pour l'homme.

Ce n'est donc pas son statut d'individu substantifié qui fait de l'être humain une personne, c'est au contraire son caractère relatif et déterminé et surtout la manière dont il est uni à la Nature et aux autres hommes qui le font accéder à la dignité de personne. Il est permis de constater ici un renversement des valeurs par rapport à la conception traditionnelle de la personne, ce n'est pas parce qu'il existe par soi et pour soi que l'homme est une personne, c'est à l'inverse parce qu'il est lié, par des liens qui lui sont spécifiques, à la nature et donc à l'humanité tout entière dont il est solidaire qu'il acquiert le statut et la valeur de personne.

Et comme toute valeur s'enracine dans le désir, puisque ce n'est pas parce qu'une chose a de la valeur que nous la désirons, mais parce que nous la désirons qu'elle a de la valeur ${ }^{37}$, cette dignité de l'homme se fonde sur le désir qu'a l'homme libre de partager sa liberté avec tous les autres hommes. C'est pourquoi l'homme libre aime les hommes, car il a conscience que leur existence contribue à l'accroissement de sa perfection et lui procure de la joie, et c'est cet amour de l'homme pour l'homme qui fonde la dignité humaine.

Ainsi le projet énoncé par Spinoza dans le Traité de la réforme de l'entendement peut-il être interprété comme un plaidoyer pour l'affirmation de la dignité de la personne humaine. Il y expose précisément ce qui distingue l'homme des autres parties de la Nature. Il explique comment l'homme peut accéder à une nature supérieure par la connaissance, ce qui aura pour conséquence concrète le progrès des sciences et des techniques, ainsi qu'un progrès moral et politique créant les conditions pour que les hommes puissent vivre plus dignement.

37. Éthique, Troisième partie, scolie de la proposition IX. 